УДК 511.176

R. Frontczak, T. Goy

\title{
GENERAL INFINITE SERIES EVALUATIONS INVOLVING FIBONACCI NUMBERS AND THE RIEMANN ZETA FUNCTION
}

\begin{abstract}
R. Frontczak, T. Goy. General infinite series evaluations involving Fibonacci numbers and the Riemann zeta function, Mat. Stud. 55 (2021), 115-123.

The purpose of this paper is to present closed forms for various types of infinite series involving Fibonacci (Lucas) numbers and the Riemann zeta function at integer arguments. To prove our results, we will apply some conventional arguments and combine the Binet formulas for these sequences with generating functions involving the Riemann zeta function and some known series evaluations. Among the results derived in this paper, we will establish that

where $\gamma$ is the familiar Euler-Mascheroni constant.

$$
\sum_{k=1}^{\infty}(\zeta(2 k+1)-1) F_{2 k}=\frac{1}{2}, \quad \sum_{k=1}^{\infty}(\zeta(2 k+1)-1) \frac{L_{2 k+1}}{2 k+1}=1-\gamma
$$
\end{abstract}

1. Motivation and introduction. This paper is devoted to combine two very popular and important mathematical objects: the Riemann zeta function and Fibonacci numbers. Both objects have been studied intensively and are well understood but identities connecting them are not documented in the mathematical literature. This is somewhat unexpected and surprising. In this article, we attempt to fill this gap. More precisely, we will study some classes of infinite series involving these two famous mathematical objects. Using generating functions combined with some fairly routine arguments, we will be able to express the series in closed form. Surprisingly, in most cases the infinite series will possess simple analytical expressions, many of them involving trigonometric functions.

Recall that the Riemann zeta function $\zeta(s), s \in \mathbb{C}$, is defined by [1]

$$
\zeta(s)=\sum_{k=1}^{\infty} \frac{1}{k^{s}}, \quad \operatorname{Re}(s)>1 .
$$

The analytical continuation to all $s \in \mathbb{C}$ with $\operatorname{Re}(s)>0, s \neq 1$, is given by

$$
\zeta(s)=\left(1-2^{1-s}\right)^{-1} \sum_{k=1}^{\infty} \frac{(-1)^{k+1}}{k^{s}} .
$$

The evaluation of $\zeta(s)$ at integer arguments is an old problem that still challenges the mathematical community. For even positive integer arguments this problem was completely solved by Euler showing that

$$
\zeta(2 n)=(-1)^{n+1} \frac{(2 \pi)^{2 n}}{2(2 n) !} B_{2 n}
$$

2010 Mathematics Subject Classification:11B39, 11M06, 40C15.

Keywords: Fibonacci number; Lucas number; Riemann zeta function; digamma function; generating function. doi:10.30970/ms.55.2.115-123

(C) R. Frontczak, T. Goy, 2021 
where $B_{n}$ are the Bernoulli numbers [2]. For odd integer arguments, the problem is still open. Much more information about $\zeta(s)$ is contained in the textbooks $[4,10]$, among others.

On the other hand, the Fibonacci number sequence is one of the most famous integer sequences in the mathematical world. The Fibonacci numbers $F_{n}$ and the companion sequence of Lucas numbers $L_{n}$ are defined for $n \geq 0$ as $F_{n+2}=F_{n+1}+F_{n}$ and $L_{n+2}=L_{n+1}+L_{n}$ with initial conditions $F_{0}=0, F_{1}=1, L_{0}=2$ and $L_{1}=1$, respectively. The Binet formulas are given by

$$
F_{n}=\frac{\alpha^{n}-\beta^{n}}{\alpha-\beta}, \quad L_{n}=\alpha^{n}+\beta^{n}
$$

where $\alpha$ is the golden ratio, i.e., $\alpha=\frac{1+\sqrt{5}}{2}$ and $\beta=-\frac{1}{\alpha}=\frac{1-\sqrt{5}}{2}$. The sequences $\left(F_{n}\right)_{n \geq 0}$ and $\left(L_{n}\right)_{n \geq 0}$ possess many interesting properties and appear in mathematical branches such as combinatorics and graph theory. See [8] for more details. They are indexed in the On-Line Encyclopedia of Integer Sequences [11] with entries A000045 and A000032, respectively.

It is well known that every real number has representation of the form $\sum_{k=0}^{\infty} \zeta(k) a_{k}$ with $\left(a_{k}\right)_{k \geq 0}$ rational [3]. We focus on the cases where $a_{k}$ is a some function of $F_{k}$ or $L_{k}$. Infinite series evaluations involving Fibonacci (Lucas) numbers and the zeta function are rare. Very recently, the following evaluations involving the Riemann zeta function at positive even integer argument and scaled even Fibonacci (Lucas) numbers were stated in [5]:

$$
\sum_{k=1}^{\infty} \zeta(2 k) \frac{F_{2 k}}{5^{k}}=\frac{\pi}{2 \sqrt{5}} \tan \frac{\pi}{2 \sqrt{5}}, \quad \sum_{k=1}^{\infty} \zeta(2 k) \frac{L_{2 k}}{5^{k}}=\frac{\pi}{2 \sqrt{5}} \tan \frac{\pi}{2 \sqrt{5}}+1 .
$$

A still more appealing identity involving $\zeta(s)$ at odd integer argument and Fibonacci numbers comes as another problem proposal from [6]:

$$
\sum_{k=1}^{\infty} \zeta(2 k+1) \frac{F_{2 k}}{5^{k}}=\frac{1}{2}
$$

Interestingly, the Lucas counterpart does not possess such a nice structure:

$$
\sum_{k=1}^{\infty} \zeta(2 k+1) \frac{L_{2 k}}{5^{k}}=\frac{3}{2}-2 \sum_{n=1}^{\infty} \frac{1}{n\left(5 n^{2}-5 n+1\right)\left(5 n^{2}+5 n+1\right)} .
$$

A few more such relations can be found in [7].

The goal of this article is to continue the research in this direction and to present more closed forms for some types of infinite series involving Fibonacci (Lucas) numbers and the Riemann zeta function. To prove the results, we will mainly work with generating functions and some series evaluations. In addition, in some of our proofs we will apply properties of the digamma function $\psi(z), z \in \mathbb{C}$. Recall that $\psi(z)$ is the first logarithmic derivative of the gamma function $\Gamma(z)$ (the Euler integral of the second kind) [1], i.e.,

$$
\psi(z)=(\ln \Gamma(z))^{\prime}=\frac{\Gamma^{\prime}(z)}{\Gamma(z)} .
$$

The digamma function possesses the following properties:

$$
\begin{gathered}
\psi(z+1)=\psi(z)+\frac{1}{z} \\
\psi(z+1)=-\gamma+\sum_{n=1}^{\infty}\left(\frac{1}{n}-\frac{1}{n+z}\right), \quad z \neq-1,-2, \ldots,
\end{gathered}
$$

and the reflection property

$$
\psi(1-z)-\psi(z)=\pi \cot \pi z,
$$


where $\gamma$ is the Euler-Mascheroni constant given by

$$
\gamma=\lim _{n \rightarrow \infty}\left(\sum_{k=1}^{n} \frac{1}{k}-\ln n\right)=0,5772156649 \ldots
$$

These properties will be employed in some of the proofs below.

2. Main results. Our first result are the following evaluations involving the Riemann zeta function at even integer arguments and Fibonacci (Lucas) numbers.

Theorem 1. For $m \geq 0$, we have

$$
\begin{aligned}
\sum_{k=1}^{\infty}(\zeta(2 k)-1) F_{2 k+m-1} & =\frac{\pi}{2 \sqrt{5}} \tan \frac{\sqrt{5} \pi}{2} L_{m}+\frac{F_{m+2}}{2} \\
\sum_{k=1}^{\infty}(\zeta(2 k)-1) L_{2 k+m-1} & =\frac{\sqrt{5} \pi}{2} \tan \frac{\sqrt{5} \pi}{2} F_{m}+\frac{L_{m+2}}{2} .
\end{aligned}
$$

Proof. From [10, p. 281] we know that

This gives with $z=\alpha$

$$
\sum_{k=1}^{\infty}(\zeta(2 k)-1) z^{2 k-1}=-\frac{\pi}{2} \cot \pi z+\frac{3 z^{2}-1}{2 z\left(z^{2}-1\right)}, \quad|z|<2 .
$$

$$
\sum_{k=1}^{\infty}(\zeta(2 k)-1) \alpha^{2 k-1}=-\frac{\pi}{2} \cot \pi \alpha+\frac{\alpha^{2}}{2},
$$

where we have used that $3 \alpha+2=\alpha^{4}$ and $\alpha^{2}=\alpha+1$. Hence,

In the same way, we get

$$
\sum_{k=1}^{\infty}(\zeta(2 k)-1) \alpha^{2 k+m-1}=-\frac{\pi}{2} \alpha^{m} \cot \pi \alpha+\frac{\alpha^{m+2}}{2} .
$$

$$
\sum_{k=1}^{\infty}(\zeta(2 k)-1) \beta^{2 k+m-1}=-\frac{\pi}{2} \beta^{m} \cot \pi \beta+\frac{\beta^{m+2}}{2} .
$$

Combining these equations according to the Binet formula and making use of the fact that $\cot (\pi / 2-x)=\tan x$ we obtain

$$
\begin{gathered}
\sum_{k=1}^{\infty}(\zeta(2 k)-1) F_{2 k+m-1}=\frac{\pi}{2 \sqrt{5}}\left(\beta^{m} \cot \pi \beta-\alpha^{m} \cot \pi \alpha\right)+\frac{F_{m+2}}{2}= \\
=\frac{\pi}{2 \sqrt{5}}\left(\beta^{m} \tan \frac{\sqrt{5} \pi}{2}+\alpha^{m} \tan \frac{\sqrt{5} \pi}{2}\right)+\frac{F_{m+2}}{2}
\end{gathered}
$$

and the proof of (4) is completed. The identity (5) is proved similarly and omitted.

Explicit examples for $m=0$ and $m=1$ are

$$
\begin{gathered}
\sum_{k=1}^{\infty}(\zeta(2 k)-1) F_{2 k-1}=\frac{\pi}{\sqrt{5}} \tan \frac{\sqrt{5} \pi}{2}+\frac{1}{2}, \quad \sum_{k=1}^{\infty}(\zeta(2 k)-1) L_{2 k-1}=\frac{3}{2}, \\
\sum_{k=1}^{\infty}(\zeta(2 k)-1) F_{2 k}=\frac{\pi}{2 \sqrt{5}} \tan \frac{\sqrt{5} \pi}{2}+1, \quad \sum_{k=1}^{\infty}(\zeta(2 k)-1) L_{2 k}=\frac{\sqrt{5} \pi}{2} \tan \frac{\sqrt{5} \pi}{2}+2 .
\end{gathered}
$$

Remark 1. We point out that instead of proving (5) directly (as we did implicitly), it can also be deduced from (4) using Fibonacci-Lucas relations $5 F_{n}=L_{n+1}+L_{n-1}$ and $L_{n}=$ $F_{n+1}+F_{n-1}$. 
A proof comparable to the one given for Theorem 1 yields the following series:

$$
\begin{gathered}
\sum_{k=1}^{\infty} \frac{\zeta(2 k)-1}{n^{2 k-1}} F_{2 k-1}=\frac{1}{\sqrt{5}} \cdot \frac{\pi \sin \frac{\pi \sqrt{5}}{n}}{\cos \frac{\pi \sqrt{5}}{n}-\cos \frac{\pi}{n}}+\frac{2 n\left(n^{4}-5 n^{2}+3\right)}{\left(2 n^{2}-3+\sqrt{5}\right)\left(2 n^{2}-3-\sqrt{5}\right)}, \\
\sum_{k=1}^{\infty} \frac{\zeta(2 k)-1}{n^{2 k-1}} L_{2 k-1}=\frac{\pi \sin \frac{\pi}{n}}{\cos \frac{\pi}{n}-\cos \frac{\pi \sqrt{5}}{n}}-\frac{2 n\left(n^{4}-n^{2}+3\right)}{\left(2 n^{2}-3+\sqrt{5}\right)\left(2 n^{2}-3-\sqrt{5}\right)} .
\end{gathered}
$$

As could be expected, the formula including the odd zeta values is more involved and possesses a semi-closed form.

Theorem 2. For $m \geq 0$, we have

$$
\begin{aligned}
& \sum_{k=1}^{\infty}(\zeta(2 k+1)-1) F_{2 k+m}=\frac{F_{m+1}}{2}+F_{m}\left(\frac{1}{2}+\frac{\pi}{2} \tan \frac{\sqrt{5} \pi}{2}-\psi(\alpha)-\gamma\right) \\
& \sum_{k=1}^{\infty}(\zeta(2 k+1)-1) L_{2 k+m}=\frac{L_{m+1}}{2}+L_{m}\left(\frac{1}{2}+\frac{\pi}{2} \tan \frac{\sqrt{5} \pi}{2}-\psi(\alpha)-\gamma\right) .
\end{aligned}
$$

Proof. From [10, p. 280] we have the following generating function:

$$
\sum_{k=1}^{\infty}(\zeta(2 k+1)-1) z^{2 k}=(1-\gamma)-\frac{1}{2}(\psi(2+z)+\psi(2-z)), \quad|z|<2 .
$$

This relation combined with the Binet formula and $2-\alpha=\alpha^{-2}=\beta^{2}, 2-\beta=\alpha^{2}$ yields

$$
\begin{gathered}
\sum_{k=1}^{\infty}(\zeta(2 k+1)-1) F_{2 k+m}= \\
=(1-\gamma) F_{m}+\frac{1}{2 \sqrt{5}}\left(\beta^{m} \psi\left(\beta^{2}+1\right)-\alpha^{m} \psi\left(\beta^{2}\right)\right)-\frac{1}{2 \sqrt{5}}\left(\alpha^{m} \psi\left(\alpha^{2}+1\right)-\beta^{m} \psi\left(\alpha^{2}\right)\right) .
\end{gathered}
$$

Now, we note that

$$
\beta^{m} \psi\left(\beta^{2}+1\right)-\alpha^{m} \psi\left(\beta^{2}\right)=L_{m}\left(\psi\left(\beta^{2}+1\right)-\psi\left(\beta^{2}\right)\right)-\alpha^{m} \psi\left(\beta^{2}+1\right)+\beta^{m} \psi\left(\beta^{2}\right)
$$

and

$$
\alpha^{m} \psi\left(\alpha^{2}+1\right)-\beta^{m} \psi\left(\alpha^{2}\right)=L_{m}\left(\psi\left(\alpha^{2}+1\right)-\psi\left(\alpha^{2}\right)\right)-\beta^{m} \psi\left(\alpha^{2}+1\right)+\alpha^{m} \psi\left(\alpha^{2}\right) .
$$

Gathering terms and keeping in mind property (2) we arrive at

$$
\sum_{k=1}^{\infty}(\zeta(2 k+1)-1) F_{2 k+m}=(1-\gamma) F_{m}+\frac{L_{m}}{2}+\frac{S_{1}+S_{2}}{2 \sqrt{5}},
$$

with $S_{1}=\beta^{m} \psi\left(\beta^{2}\right)-\alpha^{m} \psi\left(\alpha^{2}\right), S_{2}=\beta^{m} \psi\left(\alpha^{2}+1\right)-\alpha^{m} \psi\left(\beta^{2}+1\right)$.

Next, we apply property (3) to get

$$
\begin{gathered}
S_{1}=\gamma \sqrt{5} F_{m}+\sum_{n=1}^{\infty}\left(\frac{\beta^{m}}{n}-\frac{\beta^{m}}{n+\beta}-\frac{\alpha^{m}}{n}+\frac{\alpha^{m}}{n+\alpha}\right)= \\
=\gamma \sqrt{5} F_{m}-\sum_{n=1}^{\infty} \frac{(n-1) \sqrt{5} F_{m}+n \sqrt{5} F_{m-1}}{n(n+\alpha)(n+\beta)}= \\
=\gamma \sqrt{5} F_{m}-\sqrt{5} F_{m+1} \sum_{n=1}^{\infty} \frac{1}{n^{2}+n-1}+\sqrt{5} F_{m} \sum_{n=1}^{\infty} \frac{1}{n\left(n^{2}+n-1\right)} .
\end{gathered}
$$


Similarly for $S_{2}$,

$$
\begin{gathered}
S_{2}=\gamma \sqrt{5} F_{m}+\sum_{n=1}^{\infty} \frac{-(3 n+1) \sqrt{5} F_{m}+n \sqrt{5} F_{m+2}}{n\left(n+\alpha^{2}\right)\left(n+\beta^{2}\right)}=\gamma \sqrt{5} F_{m}+\sqrt{5} F_{m+1} \sum_{n=1}^{\infty} \frac{1}{n^{2}+3 n+1}- \\
-2 \sqrt{5} F_{m} \sum_{n=1}^{\infty} \frac{1}{n^{2}+3 n+1}-\sqrt{5} F_{m} \sum_{n=1}^{\infty} \frac{1}{n\left(n^{2}+3 n+1\right)} .
\end{gathered}
$$

Hence,

$$
\begin{gathered}
\sum_{k=1}^{\infty}(\zeta(2 k+1)-1) F_{2 k+m}=F_{m}+\frac{L_{m}}{2}-\frac{F_{m+1}}{2} \sum_{n=1}^{\infty} \frac{1}{n^{2}+n-1}+\frac{F_{m}}{2} \sum_{n=1}^{\infty} \frac{1}{n\left(n^{2}+n-1\right)}+ \\
+\left(\frac{F_{m+1}}{2}-F_{m}\right) \sum_{n=1}^{\infty} \frac{1}{n^{2}+3 n+1}-\frac{F_{m}}{2} \sum_{n=1}^{\infty} \frac{1}{n\left(n^{2}+3 n+1\right)}
\end{gathered}
$$

To simplify further, we note that (see [9])

$$
\begin{gathered}
\sum_{n=1}^{\infty} \frac{1}{n^{2}+n-1}=1+\frac{\sqrt{5} \pi}{5} \tan \frac{\sqrt{5} \pi}{2} \\
\sum_{n=1}^{\infty} \frac{1}{n\left(n^{2}+n-1\right)}=1-\gamma-\psi(\alpha)+\frac{5+\sqrt{5}}{10} \pi \tan \frac{\sqrt{5} \pi}{2} \\
\sum_{n=1}^{\infty} \frac{1}{n^{2}+3 n+1}=\frac{\sqrt{5} \pi}{5} \tan \frac{\sqrt{5} \pi}{2}, \\
\sum_{n=1}^{\infty} \frac{1}{n\left(n^{2}+3 n+1\right)}=1+\psi(\alpha)+\gamma-\frac{5+3 \sqrt{5}}{10} \pi \tan \frac{\sqrt{5} \pi}{2} .
\end{gathered}
$$

Using these series, from (8) we finally conclude that

$$
\sum_{k=1}^{\infty}(\zeta(2 k+1)-1) F_{2 k+m}=\frac{2 F_{m}-F_{m+1}+L_{m}}{2}+\frac{\pi F_{m}}{2} \tan \frac{\sqrt{5} \pi}{2}-F_{m}(\psi(\alpha)+\gamma) .
$$

Taking into account $L_{m}=F_{m-1}+F_{m+1}$, we have (6). The statement (7) can be proved either analogously or using the relations from Remark 1.

When $m=0$, then from (6) we get the expressions

$$
\sum_{k=1}^{\infty}(\zeta(2 k+1)-1) F_{2 k}=\frac{1}{2}, \quad \sum_{k=1}^{\infty}(\zeta(2 k+1)-1) L_{2 k}=\frac{3}{2}+\pi \tan \frac{\sqrt{5} \pi}{2}-2 \psi(\alpha)-2 \gamma
$$

In view of (1) we arrive at the beautiful result

$$
\sum_{k=1}^{\infty} \zeta(2 k+1) \frac{F_{2 k}}{5^{k}}=\sum_{k=1}^{\infty}(\zeta(2 k+1)-1) F_{2 k}=\frac{1}{2}
$$

The next result generalizes an identity from [7]. 
Theorem 3. For $m \geq 0$, we have

$$
\begin{aligned}
& \sum_{k=2}^{\infty}(\zeta(k)-1) F_{k+m-1}=F_{m+2}+\left(\frac{\sqrt{5}}{5} F_{m-1}+\frac{5+\sqrt{5}}{10} F_{m}\right) \pi \tan \frac{\sqrt{5} \pi}{2}-F_{m}(\psi(\alpha)+\gamma) \\
& \sum_{k=2}^{\infty}(\zeta(k)-1) L_{k+m-1}=L_{m+2}+\left(\frac{\sqrt{5}}{5} L_{m-1}+\frac{5+\sqrt{5}}{10} L_{m}\right) \pi \tan \frac{\sqrt{5} \pi}{2}-L_{m}(\psi(\alpha)+\gamma)
\end{aligned}
$$

Proof. Here, we work with the generating function

$$
\sum_{k=2}^{\infty}(\zeta(k)-1) z^{k-1}=1-\gamma-\psi(2-z), \quad|z|<2,
$$

which also comes from [10, p. 280]. This gives

$$
\sum_{k=2}^{\infty}(\zeta(k)-1) F_{k+m-1}=(1-\gamma) F_{m}+\frac{1}{\sqrt{5}}\left(\beta^{m} \psi(\alpha+1)-\alpha^{m} \psi(\beta+1)\right) .
$$

The remainder of the proof is as above and we leave it as an exercise.

When $m=0$, we use the Fibonacci relation $F_{-n}=(-1)^{n+1} F_{n}$ to see that

$$
\sum_{k=2}^{\infty}(\zeta(k)-1) F_{k-1}=1+\frac{\pi}{\sqrt{5}} \tan \frac{\sqrt{5} \pi}{2},
$$

which appears in [7].

3. Further related series. In this section we study some series that are closely related to the series form the last section.

Theorem 4. Let $m \geq 0$. Then we have

$$
\begin{gathered}
\sum_{k=1}^{\infty}(\zeta(2 k)-1) \frac{F_{2 k+m-1}}{k}=F_{m-1} \ln (\pi \csc \pi \alpha)+\frac{2 L_{m-1} \ln \alpha}{\sqrt{5}} \\
\sum_{k=1}^{\infty}(\zeta(2 k)-1) \frac{L_{2 k+m-1}}{k}=L_{m-1} \ln (\pi \csc \pi \alpha)+2 \sqrt{5} F_{m-1} \ln \alpha
\end{gathered}
$$

Proof. We work with the following generating function from [10, p. 281]

$$
\sum_{k=1}^{\infty}(\zeta(2 k)-1) \frac{z^{2 k}}{k}=\ln \left(\pi z\left(1-z^{2}\right) \csc \pi z\right), \quad|z|<2 .
$$

This relation yields straightforwardly for $m \geq 0$

$$
\sum_{k=1}^{\infty}(\zeta(2 k)-1) \frac{F_{2 k+m-1}}{k}=\frac{\alpha^{m-1} \ln \left(\pi \alpha^{2} \sec \pi \alpha\right)-\beta^{m-1} \ln \left(\pi \beta^{2} \sec \pi \beta\right)}{\sqrt{5}} .
$$

The first expression is obtained by simplification using $\sin \pi \alpha=\sin \pi \beta=\cos \frac{\sqrt{5} \pi}{2}$. The proof of the second expression is similar. 
When $m=0$ and $m=1$, then with the use of $L_{-n}=(-1)^{n} L_{n}$

$$
\begin{gathered}
\sum_{k=1}^{\infty}(\zeta(2 k)-1) \frac{F_{2 k-1}}{k}=\ln (\pi \csc \pi \alpha)-\frac{2 \ln \alpha}{\sqrt{5}}, \\
\sum_{k=1}^{\infty}(\zeta(2 k)-1) \frac{L_{2 k-1}}{k}=-\ln (\pi \csc \pi \alpha)+2 \sqrt{5} \ln \alpha, \\
\sum_{k=1}^{\infty}(\zeta(2 k)-1) \frac{F_{2 k}}{k}=\frac{4 \ln \alpha}{\sqrt{5}}, \quad \sum_{k=1}^{\infty}(\zeta(2 k)-1) \frac{L_{2 k}}{k}=2 \ln (\pi \sec \pi \alpha) .
\end{gathered}
$$

Theorem 5. Let $m \geq 0$. Then we have

$$
\begin{gathered}
\sum_{k=1}^{\infty}(\zeta(2 k+1)-1) \frac{F_{2 k+m}}{2 k+1}=(1-\gamma) F_{m}+\frac{L_{m-1}}{2 \sqrt{5}}(\ln (\pi \csc \pi \alpha)-2 \ln \Gamma(\alpha)-4 \ln \alpha), \\
\sum_{k=1}^{\infty}(\zeta(2 k+1)-1) \frac{L_{2 k+m}}{2 k+1}=(1-\gamma) L_{m}+\frac{\sqrt{5} F_{m-1}}{2}(\ln (\pi \csc \pi \alpha)-2 \ln \Gamma(\alpha)-4 \ln \alpha)
\end{gathered}
$$

where $\gamma$ is the Euler-Mascheroni constant.

Proof. The following generating function is stated in [10, p. 280]

$$
\sum_{k=1}^{\infty}(\zeta(2 k+1)-1) \frac{z^{2 k+1}}{2 k+1}=(1-\gamma) z+\frac{1}{2} \ln \frac{\Gamma(2-z)}{\Gamma(2+z)}, \quad|z|<2 .
$$

This relation yields straightforwardly for $m \geq 0$

$$
\sum_{k=1}^{\infty}(\zeta(2 k+1)-1) \frac{F_{2 k+m}}{2 k+1}=(1-\gamma) F_{m}+\frac{1}{2 \sqrt{5}}\left(\alpha^{m-1} \ln \frac{\Gamma(\beta+1)}{\Gamma\left(\alpha^{2}+1\right)}-\beta^{m-1} \ln \frac{\Gamma(\alpha+1)}{\Gamma\left(\beta^{2}+1\right)}\right) .
$$

The fundamental functional equation of the gamma function, $\Gamma(z+1)=z \Gamma(z)$, yields

$$
\sum_{k=1}^{\infty}(\zeta(2 k+1)-1) \frac{F_{2 k+m}}{2 k+1}=(1-\gamma) F_{m}+\frac{1}{2 \sqrt{5}}\left(\alpha^{m-1} \ln \frac{\beta \Gamma(\beta)}{\alpha^{3} \Gamma(\alpha)}-\beta^{m-1} \ln \frac{\alpha \Gamma(\alpha)}{\beta^{3} \Gamma(\beta)}\right) .
$$

Since $\frac{\beta \Gamma(\beta)}{\alpha^{3} \Gamma(\alpha)}=\left(\frac{\alpha \Gamma(\alpha)}{\beta^{3} \Gamma(\beta)}\right)^{-1}$, we can write the last equation as follows

$$
\sum_{k=1}^{\infty}(\zeta(2 k+1)-1) \frac{F_{2 k+m}}{2 k+1}=(1-\gamma) F_{m}+\frac{L_{m-1}}{2 \sqrt{5}} \ln \left(\frac{-\Gamma(\beta)}{\alpha^{4} \Gamma(\alpha)}\right) .
$$

Finally, note that $\frac{\Gamma(\beta)}{\Gamma(\alpha)}=\frac{\pi}{\Gamma^{2}(\alpha) \sin \pi \alpha}$, where we have used $\Gamma(z) \Gamma(1-z)=\frac{\pi}{\sin \pi z}$. This completes the first proof. The other one is omitted.

The special evaluations for $m=0$ and $m=1$ are

$$
\begin{gathered}
\sum_{k=1}^{\infty}(\zeta(2 k+1)-1) \frac{F_{2 k}}{2 k+1}=\frac{1}{2 \sqrt{5}}(-\ln (\pi \csc \pi \alpha)+2 \ln \Gamma(\alpha)+4 \ln \alpha), \\
\sum_{k=1}^{\infty}(\zeta(2 k+1)-1) \frac{L_{2 k}}{2 k+1}=2(1-\gamma)+\frac{\sqrt{5}}{2}(\ln (\csc \pi \alpha)-2 \ln \Gamma(\alpha)-4 \ln \alpha), \\
\sum_{k=1}^{\infty}(\zeta(2 k+1)-1) \frac{F_{2 k+1}}{2 k+1}=1-\gamma+\frac{1}{\sqrt{5}}(\ln (\csc \pi \alpha)-2 \ln \Gamma(\alpha)-4 \ln \alpha),
\end{gathered}
$$


and the interesting identity

$$
\sum_{k=1}^{\infty}(\zeta(2 k+1)-1) \frac{L_{2 k+1}}{2 k+1}=1-\gamma
$$

Theorem 6. For $m \geq 0$, we have

$$
\sum_{k=2}^{\infty}(\zeta(k)-1) \frac{F_{k+m-1}}{k}=(1-\gamma) F_{m}-\frac{\ln \Gamma(\alpha)}{\sqrt{5}} L_{m-1}+F_{m-1} \ln \alpha+\frac{\alpha^{m-1}}{\sqrt{5}} \ln \left(\frac{\pi}{\alpha^{2}} \csc \pi \alpha\right)
$$

and

$$
\sum_{k=2}^{\infty}(\zeta(k)-1) \frac{L_{k+m-1}}{k}=(1-\gamma) L_{m}-\sqrt{5} \ln \Gamma(\alpha) F_{m-1}+L_{m-1} \ln \alpha+\alpha^{m-1} \ln \left(\frac{\pi}{\alpha^{2}} \csc \pi \alpha\right) .
$$

Proof. We can prove the statements using [10, p. 280]

$$
\sum_{k=2}^{\infty}(\zeta(k)-1) \frac{z^{k}}{k}=(1-\gamma) z+\ln \Gamma(2-z), \quad|z|<2 .
$$

In case of Fibonacci numbers, we can derive

$$
\sum_{k=2}^{\infty}(\zeta(k)-1) \frac{F_{k+m}}{k}=(1-\gamma) F_{k+m}+\frac{1}{\sqrt{5}}\left(\alpha^{m} \ln (\beta \Gamma(\beta))-\beta^{m} \ln (\alpha \Gamma(\alpha))\right),
$$

from which the statement is obtained by simplification. The Lucas series is obtained analogously.

When $m=1$, then the special cases are

$$
\begin{gathered}
\sum_{k=2}^{\infty}(\zeta(k)-1) \frac{F_{k}}{k}=1-\gamma-\frac{2}{\sqrt{5}}(\Gamma(\alpha)+\ln \alpha)+\frac{1}{\sqrt{5}} \ln (\pi \csc \pi \alpha), \\
\sum_{k=2}^{\infty}(\zeta(k)-1) \frac{L_{k}}{k}=1-\gamma+\ln (\pi \csc \pi \alpha) .
\end{gathered}
$$

4. Concluding remarks. In this paper we presented new closed forms for some types of infinite series involving Fibonacci and Lucas numbers with the Riemann zeta function of integer arguments. To prove our results, we applied some routine arguments, combining Binet's formulas with generating functions and some known series evaluations. Using similar techniques, we can establish series evaluations involving the Riemann zeta function of integer arguments with Fibonacci and Lucas polynomials and other known number and polynomial sequences. For example, the following identities hold:

$$
\begin{gathered}
\sum_{k=1}^{\infty}(\zeta(2 k)-1) U_{2 k-2}(x)=\frac{2-x^{2}}{2\left(x^{2}-1\right)}+\frac{\pi}{2(\omega-\sigma)} \cdot \frac{\sin (\omega-\sigma) \pi}{\sin \omega \pi \cdot \sin \sigma \pi} \\
\sum_{k=1}^{\infty}(\zeta(2 k)-1) T_{2 k-1}(x)=\frac{x}{2}-\frac{\pi}{4} \cdot \frac{\sin (\omega+\sigma) \pi}{\sin \omega \pi \cdot \sin \sigma \pi}
\end{gathered}
$$

where $T_{n}(x)$ and $U_{n}(x)$ are Chebyshev polynomials of the first and second kind, respectively, $\omega=x+\sqrt{x^{2}-1}, \sigma=x-\sqrt{x^{2}-1}$ with $|x| \in\left(1 ; \frac{5}{4}\right)$.

5. Acknowledgments. The authors thank the anonymous referees for a careful reading of the manuscript and their helpful comments. 


\section{REFERENCES}

1. M. Abramowitz, I.A. Stegun, Handbook of Mathematical Functions with Formulas, Graphs, and Mathematical Tables, Dover, 1972.

2. T. Arakawa, T. Ibukiyama, M. Kaneko, Bernoulli Numbers and Zeta Functions, Springer, 2014.

3. J.M. Borwein, D.M. Bradley, R.E. Crandall, Computational strategies for the Riemann zeta function, J. Comput. Appl. Math., 121 (2000), 247-296.

4. H.M. Edwards, Riemann's Zeta Function, Academic Press, 1974.

5. R. Frontczak, Problem B-1267, Fibonacci Quart., 58 (2020), №2, 179.

6. R. Frontczak, Problem H-859, Fibonacci Quart., 58 (2020), №3, 281.

7. R. Frontczak, Infinite series involving Fibonacci numbers and the Riemann zeta function, Notes Number Theory Discrete Math., 26 (2020), №2, 159-166. doi: 10.7546/nntdm.2020.26.2.159-166

8. T. Koshy, Fibonacci and Lucas Numbers with Applications, Wiley, 2017.

9. A.P. Prudnikov, Yu.A. Brychkov, O.I. Marichev, Integrals and Series. Vol. 1. Elementary Functions, Gordon \& Breach Sci. Publ., 1986.

10. H.M. Srivastava, J. Choi, Zeta and q-Zeta Functions and Associated Series and Integrals, Elsevier, 2012. 11. N.J.A. Sloane, The On-Line Encyclopedia of Integer Sequences, https://oeis.org.

Landesbank Baden-Württemberg (LBBW)

Stuttgart, Germany

robert.frontczak@lbbw.de

Vasyl Stefanyk Precarpathian National University

Ivano-Frankivsk, Ukraine

taras.goy@pnu.edu.ua 